\title{
Shear stress improves the endothelial progenitor cell function via the CXCR7/ERK pathway axis in the coronary artery disease cases
}

Hua Zhou ${ }^{1 \dagger}$, Qiang Tu ${ }^{2,3+}$, Yan Zhang ${ }^{2}$, Hua Qiang Xie ${ }^{2}$, Qing Yun Shuai ${ }^{2}$, Xiao Chuan Huang ${ }^{2}$, Jie Fu ${ }^{2}$ and Zheng $\mathrm{CaO}^{2^{*}}$ (D)

\begin{abstract}
Background: Dysfunction in the late Endothelial Progenitor Cells (EPCS) is responsible for endothelial repair in patients with Coronary Artery Disease (CAD), and the shear stress is beneficial for EPCs function. However, the impact of shear stress on the capacity of EPCs in CAD patients has not been elucidated yet. The C-X-C chemokine receptor 7/extracellular signalregulated kinase (CXCR7)/(ERK) pathways are identified to regulate EPCs function in CAD patients. Here, we hypothesize that shear stress upregulates the CXCR7/ERK pathways, which restore the EPCs function in CAD patients.

Methods: The human Peripheral Blood Mononuclear Cells (PBMCs) were collected from healthy adults and CAD patients and then used for EPCs cultivation. The LV-siRNA for human CXCR7 was transfected into induced EPCs isolated from the CAD patients. Meanwhile, the EPCs from CAD patients were subjected to shear stress generated by a biomimetic device. Next, the cell viability, migration, tube formation, and apoptosis were detected by CCK-8, Transwell assay, Matrigel, and flow cytometry, respectively. Also, the CXCR7/ERK pathways in human EPCs were analyzed by Western blotting and qRT-PCR.

Result: Compared to the EPCs collected from normal adults, the CAD patient-derived EPCs showed reduced in vitro vasculogenic capacity. Also, the level of CXCR7 in CAD patient-derived EPCS was significantly reduced compared to the EPCS of healthy subjects. Meanwhile, the extracellular signal-regulated kinase (ERK), which represents a CXCR7 downstream signaling pathway, had decreased phosphorylation level. The shear stress treatment augmented the CXCR7 expression and also elevated ERK phosphorylation, which is comparable to the up-regulation of CAD patient-derived EPCs function. Further, the small interfering RNA (siRNA)-mediated CXCR7 knockdown diminished the enhanced migration, adhesion, and tube formation capacity of shear stress treated CAD patient-derived EPCS.

Conclusion: Up-regulation of the CXCR7/ERK pathways by shear stress can be a promising new target in enhancing the vasculogenic ability of CAD patient-derived EPCs.
\end{abstract}

Keywords: Shear stress, Endothelial progenitor cells, Coronary artery disease

\footnotetext{
* Correspondence: caozheng908@163.com

${ }^{\dagger}$ Hua Zhou and Qiang Tu contributed equally to this work.

2Department of Cardiology, Taihe Hospital, Hubei University of Medicine,

Shiyan 442000, Hubei, China

Full list of author information is available at the end of the article
}

(c) The Author(s). 2020 Open Access This article is licensed under a Creative Commons Attribution 4.0 International License, which permits use, sharing, adaptation, distribution and reproduction in any medium or format, as long as you give appropriate credit to the original author(s) and the source, provide a link to the Creative Commons licence, and indicate if changes were made. The images or other third party material in this article are included in the article's Creative Commons licence, unless indicated otherwise in a credit line to the material. If material is not included in the article's Creative Commons licence and your intended use is not permitted by statutory regulation or exceeds the permitted use, you will need to obtain permission directly from the copyright holder. To view a copy of this licence, visit http://creativecommons.org/licenses/by/4.0/ The Creative Commons Public Domain Dedication waiver (http://creativecommons.org/publicdomain/zero/1.0/) applies to the data made available in this article, unless otherwise stated in a credit line to the data. 


\section{Background}

Coronary artery disease (CAD) is one of the frequently occurring cardiovascular diseases (CVDs) across China [1-3]. Increasing evidence has revealed that $\mathrm{CAD}$ patients suffer from abnormal endothelial structure and function [4-7]. Thus, it is vital to maintain vascular endothelial integrity to treat $\mathrm{CAD}$. The bone marrow-derived circulating endothelial progenitor cells (EPCs) play an important role in the endothelial repair process after endothelial damage is caused [810]. Unfortunately, the EPCs function is impaired in CAD patients delaying the progression of vascular endothelial repair response [11-13]. Diabetes-related vasculopathy is the main risk factor for aggravating cardiovascular burden because impaired insulin sensitivity disrupts the vascular redox balance and thereby contributes to endothelial dysfunction [14-16]. Thus, it is necessary to identify a new way to improve the EPCs function in CAD patients and reduce the occurrence of CVDs. Shear stress is a measure of nonpharmacological intervention which benefits the vascular endothelial homeostasis [17-19]. Nonetheless, the underlying mechanism by which shear stress affects the CADEPCs at the molecular level remains unclear so far. In recent years, CXC chemokine receptor 7 (CXCR7) is considered a new chemokine receptor that responds to the stromal cellderived factor 1 (SDF-1) and regulates numerous biological processes [20-22]. Based on our previous study, impaired phosphorylation of the CXCR7-mediated extracellular signal-regulated kinase (ERK) pathway is involved in CADrelated decline of EPCs function in vitro [23].

However, only little information is available to understand the relationship between shear stress and EPCs function in CAD patients. Based on the previous studies, we assumed that impairment of the vasculogenic ability of CAD-derived EPCs was associated with the CXCR7 down-regulation and reduced ERK activation. Besides, shear stress may promote the EPCs vasculogenic function in CAD patients, and this was correlated with the increase in the CXCR7/ERK signaling. Accordingly, this study aims to explore the effect of shear stress on the up-regulation of the CXCR7/ERK pathways in EPCs function of CAD patients. Our work explored the vasculogenic capacity in vitro along with the CXCR7/ERK signaling pathways in EPCs among CAD and healthy controls. Subsequently, CAD-derived EPCs were subjected to a physiological condition of shear stress using a biomimetic device and were examined in vitro to test their effects on the CXCR7 signaling pathway and their vasculogenic function.

\section{Methods}

\section{Characteristics of subjects}

Based on the coronary angiography examination, ten consecutive male outpatients recently diagnosed with CAD (determined by $\geq 50 \%$ stenosis), and ten normal controls with matched ages were recruited to this study. Patients with hypertension, an active inflammatory disorder, diabetes, cardiovascular events, malignancy, and additional cardiovascular risk factors, were excluded from this study. Table 1 displays the baseline characteristics of CAD patients and normal controls. Routine tests, such as BMI, HR, blood pressure, fasting plasma glucose, biochemical indexes, etc. were gauged and recorded. The study protocol was approved by the Ethics Committee of Taihe Hospital. Each participant provided the written informed consent before their participation.

\section{Culture of late EPCs (LEPC)}

The LEPCs were isolated and cultured according to the previous description [24]. The human-derived peripheral blood mononuclear cells (PBMCs) were inoculated into 6-well plates containing $5 \mu \mathrm{g} / \mathrm{mL}$ human fibronectin, followed by culturing in the EGM-2 medium (Promocell, USA). The cells were cultured at $37^{\circ} \mathrm{C}$ with $5 \% \mathrm{CO}_{2}$ in a humid environment. After 4 days of culture, the cells were washed with PBS to remove the non-adherent cells, and the original medium was replaced. Typically, EPCs were positive in the uptake of $0.02 \mathrm{mg} / \mathrm{mL}$ DiI-acLDL (Invitrogen, USA) and binding of $0.01 \mathrm{mg} / \mathrm{mL}$ FITCconjugated BS-1 lectin (Sigma-Aldrich, USA), according to the previous description [25]. Also, flow cytometry was done to examine the endothelial markers in the cultivated LEPCs using the following antibodies: 1. FITCconjugated monoclonal mouse anti-human antibodies that recognize CD31 (eBioscience, USA) and vWF (Novus Biologicals, USA), and 2. APC-conjugated

Table 1 The baseline subject features

\begin{tabular}{llll}
\hline Group & $\begin{array}{l}\text { Control } \\
(\boldsymbol{n}=10)\end{array}$ & $\begin{array}{l}\text { CAD patients } \\
(\boldsymbol{n}=10)\end{array}$ & $\boldsymbol{P}$ value \\
\hline Age(yrs) & $59.9 \pm 8.17$ & $64.6 \pm 6.97$ & 0.483 \\
BMI(kg/m $\left.{ }^{2}\right)$ & $22.44 \pm 1.8$ & $23.92 \pm 2.57$ & 0.391 \\
HR(bpm) & $70.1 \pm 7.64$ & $72.1 \pm 8.14$ & 0.628 \\
Systolic blood pressure $(\mathrm{mmHg})$ & $117.4 \pm 11.4$ & $118 \pm 10.8$ & 0.97 \\
Diastolic blood pressure $(\mathrm{mmHg})$ & $76.7 \pm 6.55$ & $77.7 \pm 6.86$ & 0.873 \\
Fasting plasma glucose $(\mathrm{mmol} / \mathrm{L})$ & $4.82 \pm 0.37$ & $4.91 \pm 0.49$ & 0.519 \\
Total cholesterol (mmol/L) & $4.38 \pm 0.58$ & $4.49 \pm 0.62$ & 0.5 \\
Triglicerides(mmol/L) & $1.53 \pm 0.1$ & $1.48 \pm 0.68$ & 0.335 \\
LDL cholesterol(mmol/L) & $3.11 \pm 0.34$ & $3.33 \pm 0.22$ & 0.615 \\
HDL cholesterol(mmol/L) & $1.5 \pm 0.16$ & $1.35 \pm 0.22$ & 0.161 \\
Blood urea nitrogen $(\mathrm{mmol} / \mathrm{L})$ & $4.66 \pm 0.56$ & $4.93 \pm 0.7$ & 0.384 \\
Creatinine ( $\mu$ mol/L) & $68.6 \pm 9.52$ & $73.6 \pm 11.2$ & 0.303 \\
Alanine aminotransferase $(\mathrm{U} / \mathrm{L})$ & $26.7 \pm 6.07$ & $24.6 \pm 5.74$ & 0.56 \\
Aspartate aminotransferase $(\mathrm{U} / \mathrm{L})$ & $19.8 \pm 2.04$ & $21.6 \pm 5.81$ & 0.068 \\
\hline
\end{tabular}

Data are shown as mean \pm SD

CAD coronary artery disease, $B M I$ body mass index, $H R$ heart rate, $H D L$ highdensity lipoprotein, $L D L$ low density lipoprotein 
monoclonal mouse anti-human antibodies that recognize Tie-2 (Novus Biologicals, USA). Notably, LEPCs at the third passage (approximately 4 weeks) were utilized in each of the assays. According to the separation and culture protocol, EPCs were the cells that attached and appeared spindle-shaped [26].

\section{Shear stress test}

EPCs were subjected to shear stress using the flow chamber loading device. Firstly, the EPCs were inoculated on the glass slides and subsequently put on the parallel-plate flow chamber channel. After culturing for 1 day, the hydrodynamic shear stress was applied to the adherent cells on a parallel-plate flow chamber, according to the previously depicted procedure [27]. Later, the seeded cells were exposed to 6,12 , and $24 \mathrm{~h}$ of shear stress at $0,5,15$, and $30 \mathrm{dyn} / \mathrm{cm}^{2}$, separately. Typically, the shear stress was determined according to the formula, $\mathrm{T}=6 \mathrm{Q} \mu / \mathrm{bh}$ stress, where $\mathrm{Q}$ stands for flow rate, $\mu$ is the medium viscosity, $\mathrm{b}$ is the channel width, and $\mathrm{h}$ is the channel height. While the control cells were maintained at static conditions, each experiment was carried out in an incubator at $37^{\circ} \mathrm{C}$ with $5 \% \mathrm{CO}_{2}$.

\section{Knockdown of CXCR7}

The CXCR7 level in EPCs was knocked down using the Mission lentiviral siRNA transfection particles. Viral transfection was performed according to the manufacturer protocol (GenePharma Co., Ltd., Shanghai). After culturing the cells for 28 days, they were transferred into a serum-free medium containing the CXCR7-targeting siRNA (CXCR7-siRNA) or non-targeting siRNA (scrambled-siRNA) lentiviral particles. After $6 \mathrm{~h}$ of transfection, cells were washed with PBS and incubated for $48 \mathrm{~h}$ in the EPCs medium before analysis.

\section{EPCs proliferation}

After culturing for 28 days, the human-derived EPCs were subjected to laminar shear stress in vitro for 6, 12, and $24 \mathrm{~h}$ at $0,5,15$, and $30 \mathrm{dyn} / \mathrm{cm}^{2}$, separately. EPCs or EPCs treated with shear stress were placed on the glass slides $\left(1 \times 10^{4}\right.$ cells per well) and cultured for another 6 , 12 , and $24 \mathrm{~h}$. Then, the glass slides were placed in the 24-well plates containing $200 \mu \mathrm{L}$ of fresh medium supplemented with $20 \mu \mathrm{L}$ CCK8 (Beyotime Biotechnology, China) solution, followed by $1 \mathrm{~h}$ incubation. Then, a microplate reader (Thermo Fisher, USA) was used to determine the absorbance at a wavelength of $450 \mathrm{~nm}$.

\section{EPCs migration test}

The modified Boyden chamber (Millipore, USA) was adapted to determine the migratory capacity of EPCs. $5 \times 10^{4}$ EPCs were resuspended into $250 \mu \mathrm{L}$ of serumfree EBM-2 medium and pipetted onto the upper chamber. Later, this chamber was placed into a 24-well dish supplemented with $500 \mu \mathrm{L}$ EBM-2 medium containing $10 \% \mathrm{FBS}$. After incubating for $8 \mathrm{~h}$ at $37^{\circ} \mathrm{C}$, investigators, who were blind to the treatment grouping counted the transmigrated cells independently.

\section{EPCs adhesion test}

$3 \times 10^{4}$ EPCs were resuspended into $300 \mu \mathrm{L}$ of EBM-2 medium and pipetted onto a 24-well plate coated with human fibronectin $(5 \mu \mathrm{g} / \mathrm{mL})$ and incubated at $37^{\circ} \mathrm{C}$ for $3 \mathrm{~h}$. Later, the 24-well plate was washed with PBS to remove the non-adherent cells, while the adherent cells were fixed with $4 \%$ paraformaldehyde and stained using $0.1 \%$ crystal violet. Finally, the investigators blind to the treatment grouping counted the adherent cells independently.

\section{Tube formation test for EPCs}

The growth factor-depleted Matrigel (BD) was thawed overnight at $4{ }^{\circ} \mathrm{C}$. After complete thawing, $100 \mu \mathrm{L}$ of Matrigel was added in the 24-well plates for even distribution, followed by $2 \mathrm{~h}$ incubation at $37^{\circ} \mathrm{C} .1 \times 10^{5} \mathrm{EPCs}$ were then resuspended using $500 \mu \mathrm{L}$ of EBM-2 medium and then added onto the Matrigel top. After incubating for $4 \mathrm{~h}$ at $37^{\circ} \mathrm{C}$, each well was imaged using the microscope, and the mean tubule number was calculated based on 3-5 randomly selected fields.

\section{EPCs apoptosis assay}

The Annexin V-FITC Apoptosis Detection Kit (eBioscience, USA) was used to examine the apoptosis of EPCs. After $12 \mathrm{~h}$ of shear stress treatment, Annexin VFITC, along with propidium iodide (PI), was added to the rinsed cells at a density of $1 \times 10^{6}$ cells $/ \mathrm{mL}$ in the FACS buffer at ambient temperature for 15 mins in the dark. Next, cells were analyzed using flow cytometry.

\section{RNA isolation and real-time qRT-PCR}

The Trizol reagent (Takara, Japan) was used to extract the total RNA. The SYBR ${ }^{\circ}$ Green PCR Master Mix and iSript cDNA Synthesis Kit (Takara, Japan) were used for real-time qRT-PCR as per the manufacturer's instructions. The expression levels were corrected based on the GAPDH mRNA level and calculated according to the $2^{-\Delta \Delta} \mathrm{CT}$ approach. Specifically, the primers mentioned in Table 2 were used for the experiment.

\section{Western blotting}

The cell lysis buffer (Beyotime, China) was used to harvest the EPCs proteins. Then, SDS-PAGE was run to isolate the extracted proteins, followed by transferring the proteins onto the PVDF membranes (Millipore, USA). Typically, the rabbit anti-phospho-ERK, rabbit anti-CXCR7, and rabbit anti-ERK antibodies (all 1:1000; Abcam, USA), and the mouse anti-GAPDH antibody (1: 
Table 2 The information of $\mathrm{qPCR}$ primers

\begin{tabular}{lll}
\hline homo GAPDH & Forward & 5'-GGTGTGAACCATGAGAAGTATGA-3' \\
& Reverse & 5'-GAGTCCTTCCACGATACCAAAG-3' \\
homo CXCR7 & Forward & 5'-CTCTACACGCTCTCCTTCATTT-3'; \\
& Reverse & 5'-GTGGTCTTGGCCTGGATATT-3' \\
homo ERK & Forward & 5'-AGAGAACCCTGAGGGAGATAAA-3' \\
& Reverse & 5'-CGATGGTGGTGCTCGAATA-3' \\
\hline
\end{tabular}

5000; Abbkine, China) were used. Later, the HRPlabeled anti-rabbit IgG and anti-mouse IgG (both 1: 5000; Beyotime, China) were used for visualizing the proteins, and ECL chemiluminescence system (Tanon, China) was used for color development.

\section{Statistical analyses}

The data were presented as mean \pm standard deviation (SD). The SPSS 18.0 software was used for statistical analyses. The Kolmogorov-Smirnov test was used to study the distribution of the variables. The unpaired Student t-test or Welchs t-test (normal distribution) was employed to compare the two groups. The variance test (ANOVA) followed by the post-hoc test was used to evaluate the statistical significance of multiple groups of data with a parametric distribution. A difference of $P<$ 0.05 indicated a statistical significance.

\section{Result}

\section{Subject features}

Differences in the baseline subject features were not statistically significant in normal controls compared to the CAD cases (Table 1). Figure S1 characterizes the late EPCs.

\section{Down-regulation of CXCR7/ERK signaling pathways decreased the CAD-derived EPCs function in vitro}

The late EPCs function in vitro was assessed to investigate the effect of CXCR7/ERK signaling pathways on EPCs dysfunction in CAD cases. Compared to the normal controls, the CAD-derived EPCs had declined migration and adhension capacity (Fig. 1a-b). The tube formation test was carried out to investigate the angiogenic potential of EPCs in vitro, thus determining EPCs vasculogenic function. The tube formation capacity of late EPCs in CAD cases declined consistently and remarkably compared to that of the healthy subjects (Fig. 1c). Meanwhile, the CXCR7 and p-ERK expression decreased within the CAD-derived EPCs when compared to that of the EPCs from normal controls, as verified by Western Blotting (Fig. 1d). Thus, it was speculated that CXCR7/ERK signaling pathways played a vital role in the normal EPCs function, and also the decreased CADEPCs vasculogenic ability may be associated with the down-regulated CXCR7/ERK signaling pathways to some extent.

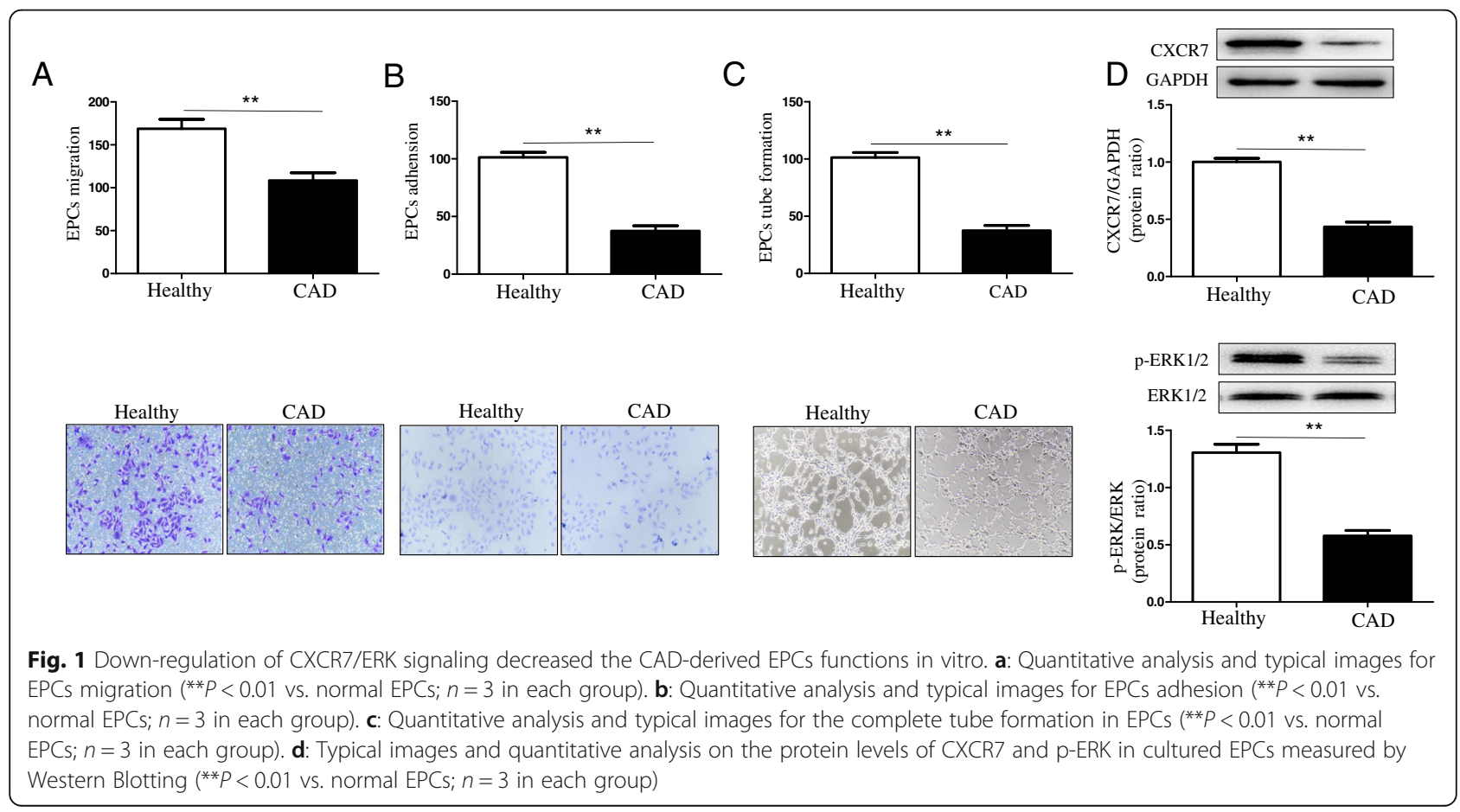



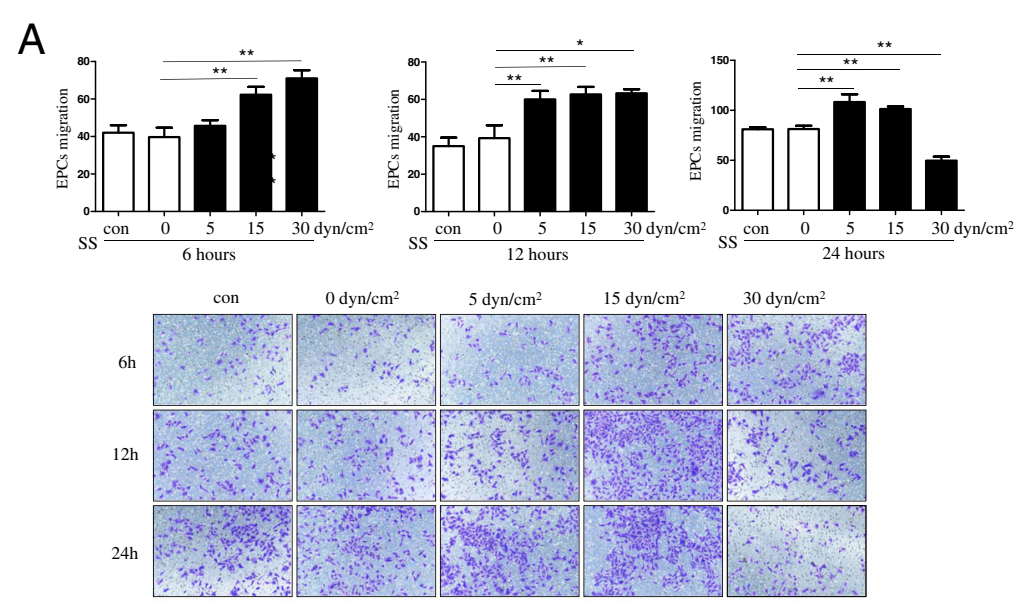

B
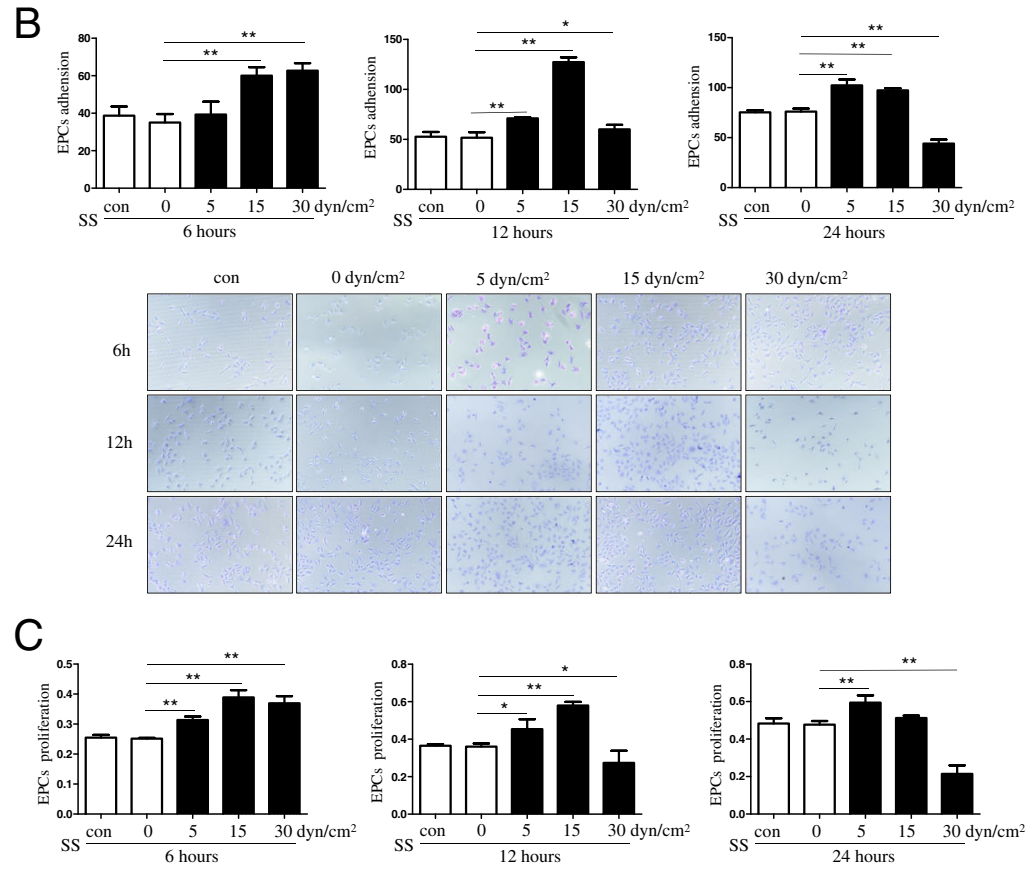

D
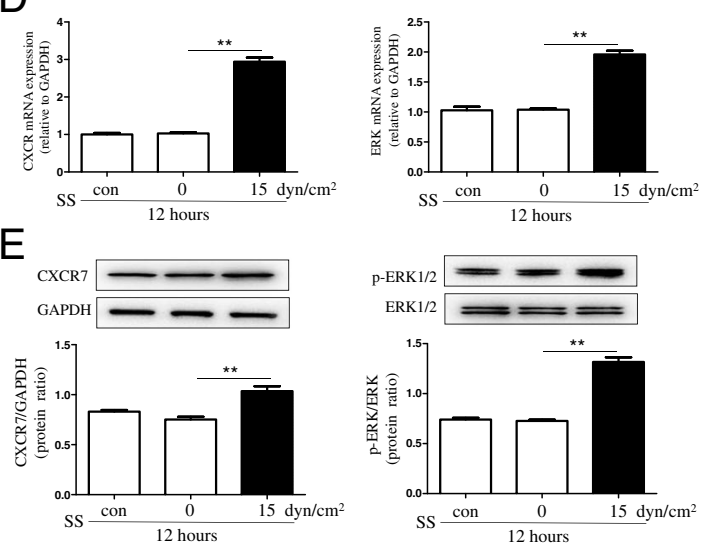

Fig. 2 (See legend on next page.) 
(See figure on previous page.)

Fig. 2 Shear stress enhanced the CAD-derived EPCs proliferation, adhesion, and migration capacities in vitro. a-c: CAD-derived EPCs were exposed to shear stress treatment for 6, 12, and $24 \mathrm{~h}$, separately, at 0,5, 15, and $30 \mathrm{dyn} / \mathrm{cm}^{2}$. a: Quantitative analysis and typical images for EPCs migration ( ${ }^{* *} P<0.01$ vs. Static CAD-EPCs; $n=3$ in each group). b: Quantitative analysis and typical images for EPCs adhesion $\left({ }^{*} p<0.05 /{ }^{* *} p<\right.$ 0.01 vs. Static CAD-EPCs; $n=3$ in each group). c: Quantitative analysis for EPCs proliferation ${ }^{*} p<0.05{ }^{* *} p<0.01 \mathrm{vs}$. Static CAD-EPCs; $n=3$ in each group). d: Quantitative analysis for the mRNA levels of CXCR7 and ERK in EPCs treated with or without $12 \mathrm{~h}$ of shear stress at $15 \mathrm{dyn} / \mathrm{cm}^{2}$ determined through Real-time PCR ( $n=3$ in each group). e: Quantitative analysis and typical images for the protein levels of CXCR7 and $p$-ERK ERK in EPCs treated with or without $12 \mathrm{~h}$ of shear stress at $15 \mathrm{dyn} / \mathrm{cm}^{2}$ measured by Western Blotting $\left({ }^{*} p<0.05 /{ }^{* *} p<0.01 \mathrm{vs}\right.$. Static CAD-EPCs; $n=$ 3 in each group)

\section{Shear stress up-regulated the CAD-derived EPCs proliferation, adhesion, and migration in vitro}

To investigate the role of shear stress on the CAD-derived EPCs function in vitro, EPCs were exposed to 6, 12, and $24 \mathrm{~h}$ shear stress at $0,5,15$, and $30 \mathrm{dyn} / \mathrm{cm}^{2}$, separately. The results suggested that shear stress significantly enhanced EPCs migration and adhesion capacity in CAD patients (Fig. 2a-b). Additionally, EPCs proliferation was promoted after being exposed to shear stress in vitro (Fig. 2c). Typically, laminar shear stress exposure at 15-dyn/ $\mathrm{cm}^{2}$ for $12 \mathrm{~h}$ significantly enhanced the CAD-derived EPCs migration, adhesion, and proliferation activities. Thus, the relationship between the enhanced EPCs vasculogenic ability after being exposed to shear stress with the CXCR7/ERK signaling pathways was explored. We found that $12 \mathrm{~h}$ of shear stress exposure in EPCs at $15-\mathrm{dyn} / \mathrm{cm}^{2}$ markedly up-regulated the CXCR7 expression at mRNA (Fig. 2d) and protein (Fig. 2e) levels. Similarly, p-ERK levels (Fig. 2d-e) were remarkably up-regulated in the EPCs that underwent shear stress treatment compared towith those cultivated at static conditions.

\section{Shear stress exposure boosted the CAD-derived EPCs ability and affected apoptosis via the CXCR7/ERK signaling}

Subsequently, the effect of shear stress-mediated upregulation of CXCR7/ERK signaling pathways in enhancing the EPCs functions was examined. According to our results, the lentiviral siRNA-induced CXCR7 silencing significantly attenuated the migratory (Fig. 3a), adhesive (Fig. 3b), and the tube forming (Fig. 3c) activities of CAD-derived EPCs on being exposed to shear stress in vitro. On the contrary, the transfection of lentiviral scrambled siRNA particles into EPCs made no difference to these functional factors. Further, the knockdown of CXCR7 declined apoptosis was mitigated by shear stress treatment in CAD patients (Fig. 3d). Overall, these findings revealed that exposure to shear stress boosted the CAD-derived EPCs vasculogenic ability through the CXCR7/ERK signaling pathways.

\section{Discussion}

The result of this study were as follows: (1) the migration, adhesion and tube formation activities of CAD- derived EPCs in vitro, remarkably decreased compared to those of the healthy subjects; (2) diminishing the CXCR7/ERK signaling pathways impaired the CADderived EPCs function in vitro; and (3) exposure to shear stress mitigated the impaired EPCs function in the CAD patients by augmenting the CXCR7/ERK pathways. Overall, our study demonstrated that the CXCR7/ERK signaling pathways played an essential role in the CADderived EPCs function, and the shear stress exposure in vitro may be a favorable method to enhance the EPCs function.

The circulating EPCs are of great importance in maintaining endothelial integrity after endothelial injury, and CAD is related to decrease EPCs number and function in the human body [12, 28-30]. In this study, the CADderived EPCs had markedly decreased abilities compared to those collected from the normal controls, suggesting that CAD decreased the endogenous vasculogenic abilities of EPCs. Emerging evidence indicates that CXCR7 plays an important role in regulating multiple cellular functions [20-22, 31]. Moreover, in the previous studies, CXCR7 regulates the EPCs proliferating, adhesive and vasculogenic activities in vitro [32, 33]. The CXCR7 signaling was closely associated with EPCs functions, and thus, we assumed that the dysregulated CXCR7 signaling may be associated with EPCs functional impairment in the CAD patients. This study suggested that the CXCR7 level was remarkably decreased in CAD-derived EPCs compared to those in normal controls, as previously reported [23]. ERK is a critical molecular signaling pathway involved in the survival, differentiation, and proliferation of various cells. Therefore, we assumed that the ERK signaling pathway participated in regulating the EPCs function mediated by CXCR7 [34-36]. For a better understanding of the relationship between CXCR7 and ERK, the phosphorylated ERK (p-ERK) level was determined, which suggested that p-ERK level remarkably decreased in CAD-derived EPCs compared to that of the EPCs from healthy subjects. According to these results, the CXCR7/ERK signaling pathways play a vital role in the EPCs function. Therefore, a novel approach that enhances the CXCR7/ERK signaling pathways can also potentially enhance the EPCs vasculogenic activities while reversing the vascular endothelial integrity and homeostasis in CAD patients. 
A

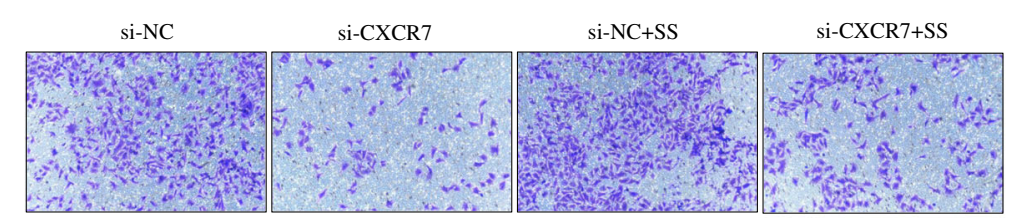

B

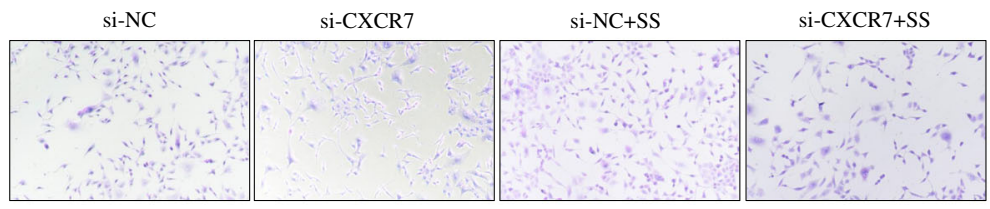

C

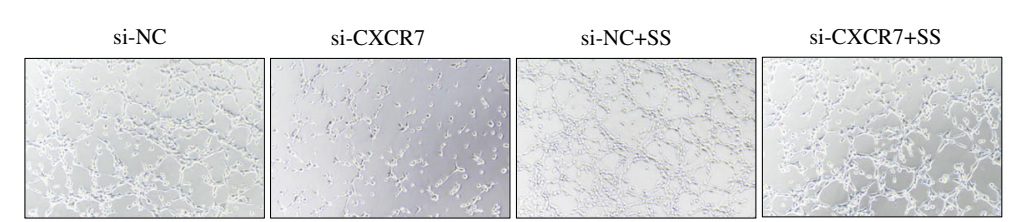

D
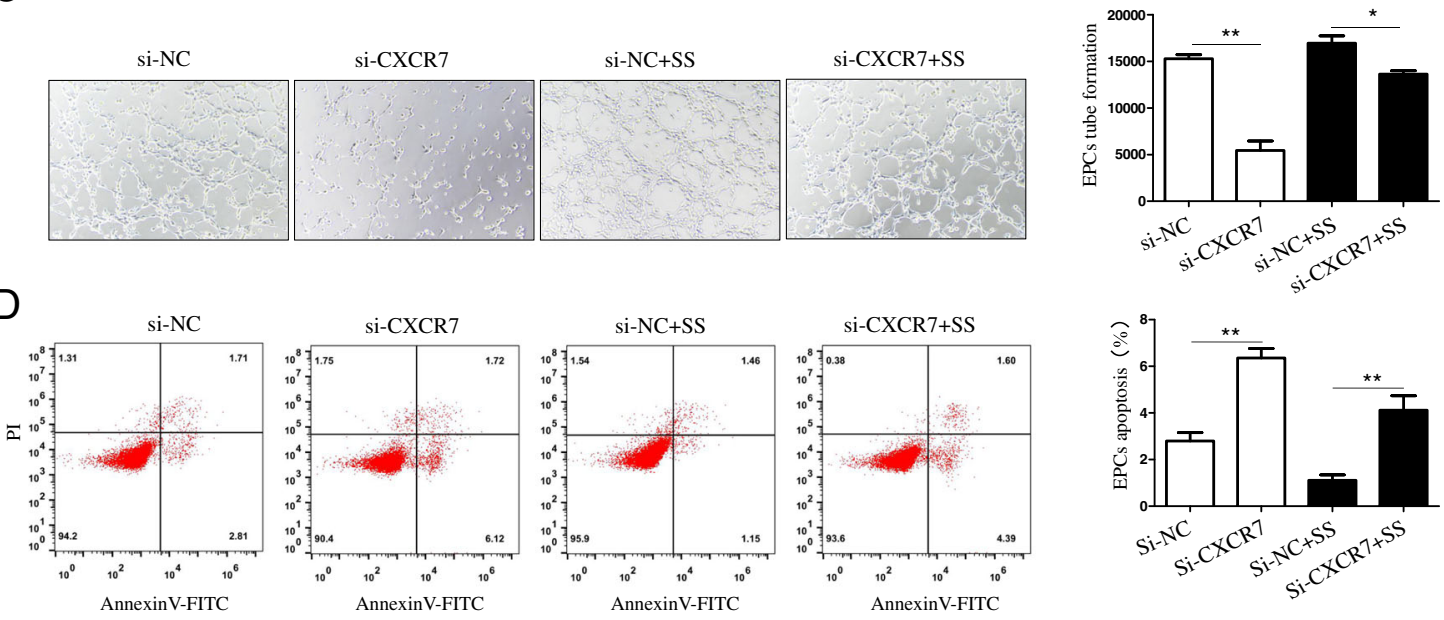

E
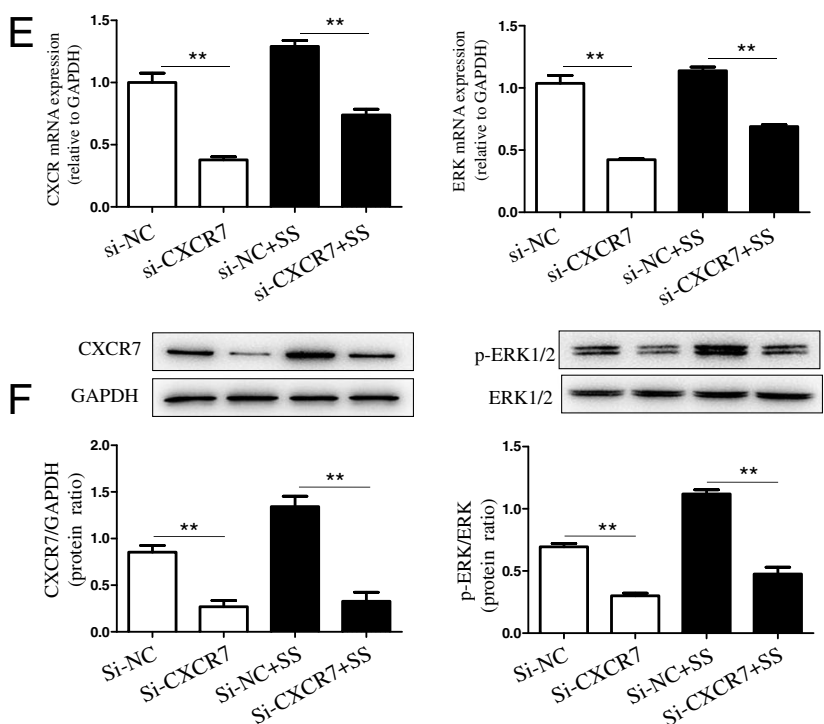

Fig. 3 (See legend on next page.) 
(See figure on previous page.)

Fig. 3 Blockade of CXCR7/ERK signaling mitigated the enhanced CAD-derived EPCs functions mediated by shear stress. a-d: The cultivated EPCs were subjected to scrambled-siRNA or Mission lentiviral CXCR7-siRNA infection for $48 \mathrm{~h}$, and another $12 \mathrm{~h}$ of shear stress at $15 \mathrm{dyn} / \mathrm{cm}^{2}$. Then, migration, adhesion, tube formation, and apoptosis assays were carried out on these cells. a: Quantitative analysis and typical images for EPCs migration ( ${ }^{*} P<0.01$ vs. siRNA-transduced CAD-EPCs in the presence or absence of shear stress treatment; $n=3$ in each group). $\mathbf{b}$ : Quantitative analysis and typical images for EPCs adhesion ${ }^{*} P<0.01$ vs. siRNA-transduced CAD-EPCs in the presence or absence of shear stress treatment; $n=3$ in each group). c: Quantitative analysis and typical images for the complete tube formation of EPCs (**P $<0.01$ vs. siRNA-transduced CADEPCs in the presence or absence of shear stress treatment; $n=3$ in each group). d: Quantitative analysis and typical images for EPCs apoptosis ( ${ }^{*} P<0.01$ vs. siRNA-transduced CAD-EPCs with or without shear stress exposure; $n=3$ in each group). e: Quantitative analysis for the CXCR7 and ERK mRNA levels in EPCs (**P $<0.01$ vs. siRNA-transduced CAD-EPCs in the presence or absence of shear stress exposure; $n=3$ in each group). $\mathbf{f}$ : Quantitative analysis and typical images for the protein levels of CXCR7 and $p$-ERK/ERK determined through Western Blotting (**P $<0.01$ vs. siRNA-transduced CAD-EPCs with or without shear stress treatment; $n=3$ in each group)

Previous studies report that shear stress plays an important role in modulating the EPCs structure and function, which indicates that it as a vital non-pharmacologic approach in regulating EPCs function [26, 37, 38]. Nonetheless, the role of shear stress in the level of CXCR7 in EPCs, as well as its correlation with endothelial protection in the CAD cases, remains unclear. This study speculated that shear stress possibly facilitated the CXCR7/ ERK signaling pathways to promote EPCs vasculogenic activities. To test this speculation, the role of shear stress in the CAD-derived EPCs proliferation, migration, and adhesion capacities in vitro was monitored, and according to our results, laminar shear stress facilitated the proliferation, adhesion, and migration of CAD-derived EPCs in vitro. Also, the shear stress upregulated the CXCR7 level and the ERK phosphorylation in EPCs of the CAD patients. Further, such augmentation of shear stress-induced CXCR7/ERK signaling pathways in CADderived EPCs conformed to the increased migratory, adhesive, and the proliferative capacities in vitro. However, the shear stress-mediated enhanced EPCs function was mitigated via CXCR7-siRNA. The above results indicate that shear stress may be favorable for modifying the CAD-derived EPCs biological phenotype.

Our results display a strong clinical significance. The dysfunction of EPCs represents the starting step, as well as a primary factor in the dismal angiogenesis of CAD [39, 40]. Clearly, our findings show that the dysfunctional properties of EPCs are associated with CAD, suggesting that CAD negatively affects the maintenance of vascular homeostasis. Typically, identifying that CAD decreases the CXCR7/ERK signaling pathways may offer molecular targets in developing a treatment to enhance the endogenous EPCs capacity. Our findings also provide evidence that shear stress may restore CXCR7 signaling and promote EPCs capacity. This presents a vital cell-based treatment to enhance EPCs function and improve vascular repairability in CAD cases. Moreover, our study also demonstrated that the blockade of CXCR7 resulted in the down-regulation of the ERK signaling pathway after the shear stress treatment, implying that the ERK pathway is involved in the angiogenesis of CAD-derived EPCs mediated by the shear stress. All in all, our work sheds more light on EPCs-related dysfunction in CAD patients offering a new strategy in the prevention and treatment of CAD.

However, several limitations are noted in this study. Firstly, only the role of shear stress in regulating the CXCR7/ERK signaling pathways, and its association with the repairability of EPCs, in vitro was examined in this study. But, an in vivo study is warranted to examine the EPCs vasculogenic ability. Secondly, this study indicated that the abnormal CXCR7 signaling in EPCs participated in the impaired CAD-derived EPCs function but, the underlying mechanism of the reduced CXCR7 level has not been well understood yet. Thirdly, this work illustrated the effects of shear stress on the phosphorylation of CXCR7/ERK signaling pathways in the EPCs, yet the precise mechanism was not clarified.

\section{Conclusion}

In summary, the results show that the shear stress enhances CAD-derived EPCs vasculogenic abilities through boosting the activation of the CXCR7/ERK signaling pathways to some extent. Based on our findings, the shear stress effect on regulating the CXCR7/ERK signaling pathways can be an underlying treatment target to repair the EPCs function in the CAD patients.

\section{Supplementary information}

Supplementary information accompanies this paper at https://doi.org/10. 1186/s12872-020-01681-0.

Additional file 1: Figure S1. Phenotypic characterization of L-EPCS.

Additional file 2: Figure S2. Analysis of CXCR7 and p-ERK expression in normal controls and CAD-EPCS.

Additional file 3: Figure S3.The effect of shear stress on CXCR7 and pERK expression in CAD-EPCS.

Additional file 4: Figure S4. Knockdown of CXCR7 significantly attenuated the $\mathrm{p}$-ERK expression of CAD-derived EPCs following shear stress in vitro.

\section{Abbreviations}

EPCs: Endothelial progenitor cells; CAD: Coronary artery disease;; CXCR7: CXC chemokine receptor 7; ERK: Extracellular signal-regulated kinase; CVDs: Cardiovascular diseases; SDF-1: Stromal cells-derived factor 1; BMI: Body mass index; HR: Heart rate; PBMCs: Peripheral blood mononuclear 
cells; FBS: Fetal bovine serum; EGM-2: Endothelial cell growth medium-2; siRNA: Small interfering RNA; qRT-PCR: Quantitative reverse transcription PCR; GAPDH: Glyceraldehyde-3-phosphate dehydrogenase; FACS: Fluorescence activated cell sorting; CD31: Platelet endothelial cell adhesion molecule; Tie2: Tyrosine-protein kinase receptor-2; vVF: Von Willebrand factor.

\section{Acknowledgments}

Not applicable.

\section{Authors' contributions}

$\mathrm{HZ}$ conceived the project, designed the experiments, and wrote the manuscript. QT performed experiments, participated in data analysis, and contributed to the discussion and editing of the manuscript. YZ, HQX, QYS, $\mathrm{XCH}$, and JF provided support with experimental techniques and performed data analysis. ZC conceived the study, designed the experiments, helped with data analysis. All authors read and approved the final manuscript.

\section{Funding}

This study was financially supported by the National Natural Science Foundation of China (NO.81771522), the Natural Science Foundation of Hubei Province (NO.2017CFB409), and the Hubei Provincial Department of Education (NO.B2019111).

\section{Availability of data and materials}

All data generated or analyzed during this study are included in this published article and its supplementary information files.

\section{Ethics approval and consent to participate}

The study protocol had gained approval from the ethical committee of Taihe Hospital. Each case had submitted the written informed consent prior to participation.

\section{Consent for publication}

Not applicable.

\section{Competing interests}

The authors declare that they have no competing interests.

\section{Author details}

${ }^{1}$ Department of Medical Ultrasound, Taihe Hospital, Hubei University of Medicine, Shiyan 442000, Hubei, China. ${ }^{2}$ Department of Cardiology, Taihe Hospital, Hubei University of Medicine, Shiyan 442000, Hubei, China. ${ }^{3}$ Hubei Key Laboratory of Embryonic Stem Cell Research, Taihe Hospital, Hubei University of Medicine, Shiyan 442000, Hubei, China.

\section{Received: 7 January 2020 Accepted: 24 August 2020}

\section{Published online: 07 September 2020}

\section{References}

1. Lv J, Yu C, Guo Y, Bian Z, Yang L, Chen Y, Tang X, Zhang W, Qian Y, Huang $Y$, et al. Adherence to healthy lifestyle and cardiovascular diseases in the Chinese population. J Am Coll Cardiol. 2017;69(9):1116-25.

2. Xiong HL, Peng M, Jiang XJ, Che WQ, Dong H, Chen Y, Zou YB, Gao RL, Liu LS. Time trends regarding the etiology of renal artery stenosis: 18 years' experience from the China Center for Cardiovascular Disease. J Clin Hypertens (Greenwich). 2018;20(9):1302-9.

3. Zhang M, Deng Q, Wang L, Huang Z, Zhou M, Li Y, Zhao Z, Zhang Y, Wang $L$. Corrigendum to "Prevalence of dyslipidemia and achievement of lowdensity lipoprotein cholesterol targets in Chinese adults: a nationally representative survey of 163,641 adults" [Int. J. Cardiol. 260 (2018) 196-203]. Int J Cardiol. 2018;267:218.

4. Asleh R, Levy AP, Levy NS, Asleh A, Goldenstein H, Segol I, Gulati R, Lerman LO, Lerman A. Haptoglobin phenotype is associated with high-density lipoprotein-bound hemoglobin content and coronary endothelial dysfunction in patients with mild nonobstructive coronary artery disease. Arterioscler Thromb Vasc Biol. 2019;39(4):774-86

5. Kitta Y, Obata JE, Nakamura T, Hirano M, Kodama Y, Fujioka D, Saito Y, Kawabata K, Sano K, Kobayashi T, et al. Persistent impairment of endothelial vasomotor function has a negative impact on outcome in patients with coronary artery disease. J Am Coll Cardiol. 2009;53(4):323-30.
6. Maessen MF, Eijsvogels TM, Hijmans-Kersten BT, Grotens A, Schreuder TH, Hopman MT, Thijssen DH. Vascular function and structure in veteran athletes after myocardial infarction. Med Sci Sports Exerc. 2017;49(1):21-8.

7. Park KH, Sun T, Diez-Delhoyo F, Liu Z, Yang SW, Lennon RJ, Herrmann J, Gulati R, Rodriguez-Porcel M, Lerman LO, et al. Association between coronary microvascular function and the vasa vasorum in patients with early coronary artery disease. Atherosclerosis. 2016;253:144-9.

8. Asahara T, Murohara T, Sullivan A, Silver M, van der Zee R, Li T, Witzenbichler B, Schatteman G, Isner JM. Isolation of putative progenitor endothelial cells for angiogenesis. Science. 1997;275(5302):964-7.

9. Wang C, Wang Q, Gao W, Zhang Z, Lou Y, Jin H, Chen X, Lei B, Xu H, Mao C. Highly efficient local delivery of endothelial progenitor cells significantly potentiates angiogenesis and full-thickness wound healing. Acta Biomater. 2018;69:156-69.

10. Mandraffino G, Saitta A. Endothelial and circulating progenitor cells: between diseases and therapies. Curr Med Chem. 2018;25(35):4476-7.

11. Morrone D, Felice F, Scatena C, De Martino A, Picoi MLE, Mancini N, Blasi S, Menicagli M, Di Stefano R, Bortolotti U, et al. Reply letter to Dr. Xu et al on role of circulating endothelial progenitor cells in the reparative mechanisms of stable ischemic myocardium. Int J Cardiol. 2018;260:21.

12. Padfield GJ, Tura-Ceide O, Freyer E, Barclay GR, Turner M, Newby DE, Mills NL. Endothelial progenitor cells, atheroma burden and clinical outcome in patients with coronary artery disease. Heart. 2013;99(11):791-8.

13. Zhang $H$, Wang LJ, Si DL, Wang C, Yang JC, Jiang P, Du C, Wang JJ. Correlation between osteocalcin-positive endothelial progenitor cells and spotty calcification in patients with coronary artery disease. Clin Exp Pharmacol Physiol. 2015;42(7):734-9.

14. Paneni F, Beckman JA, Creager MA, Cosentino F. Diabetes and vascular disease: pathophysiology, clinical consequences, and medical therapy: part I. Eur Heart J. 2013:34(31):2436-43.

15. Paneni $F$, Costantino S, Cosentino F. Role of oxidative stress in endothelial insulin resistance. World J Diabetes. 2015;6(2):326-32.

16. Vecchie A, Montecucco F, Carbone F, Dallegri F, Bonaventura A. Diabetes and vascular disease: is it all about Glycemia? Curr Pharm Des. 2019;25(29): 3112-27.

17. Ando J, Yamamoto K. Vascular mechanobiology: endothelial cell responses to fluid shear stress. Circ J. 2009;73(11):1983-92.

18. Babendreyer A, Molls $L$, Simons IM, Dreymueller D, Biller $K$, Jahr H, Denecke B, Boon RA, Bette S, Schnakenberg U, et al. The metalloproteinase ADAM15 is upregulated by shear stress and promotes survival of endothelial cells. J Mol Cell Cardiol. 2019;134:51-61.

19. Chistiakov DA, Orekhov AN, Bobryshev YV. Effects of shear stress on endothelial cells: go with the flow. Acta Physiol (Oxford). 2017;219(2):382-408.

20. Bamdad S, Khademi B, Chenari N, Taseh A, Razmkhah M. Stromal cell derived factor-1, CXCR4 and CXCR7 gene transcripts in pterygia. J Curr Ophthalmol. 2017;29(1):28-32.

21. Pluchino N, Mamillapalli R, Moridi I, Tal R, Taylor H. G-protein-coupled receptor CXCR7 is overexpressed in human and murine endometriosis. Reprod Sci. 2018;25(8):1168-74

22. Sanchez-Martin L, Sanchez-Mateos P, Cabanas C. CXCR7 impact on CXCL12 biology and disease. Trends Mol Med. 2013;19(1):12-22.

23. Cao Z, Tong X, Xia W, Chen L, Zhang X, Yu B, Yang Z, Tao J. CXCR7/p-ERKsignaling is a novel target for therapeutic Vasculogenesis in patients with coronary artery disease. PLoS One. 2016;11(9):e0161255.

24. Lin $Y$, Weisdorf DJ, Solovey A, Hebbel RP. Origins of circulating endothelial cells and endothelial outgrowth from blood. J Clin Invest. 2000;105(1):71-7.

25. Chen L, Wu F, Xia WH, Zhang YY, Xu SY, Cheng F, Liu X, Zhang XY, Wang SM, Tao J. CXCR4 gene transfer contributes to in vivo reendothelialization capacity of endothelial progenitor cells. Cardiovasc Res. 2010;88(3):462-70

26. Xia WH, Yang Z, Xu SY, Chen L, Zhang XY, Li J, Liu X, Qiu YX, Shuai XT, Tao J. Age-related decline in reendothelialization capacity of human endothelial progenitor cells is restored by shear stress. Hypertension. 2012;59(6):1225-31.

27. Frangos JA, Eskin SG, McIntire LV, Ives CL. Flow effects on prostacyclin production by cultured human endothelial cells. Science. 1985;227(4693): 1477-9.

28. Sheng ZQ, Li YF, Zheng KL, Lu HH, Xie J, Wu H, Xu B. The relationship between number and function of EPCS and concentration of VEGF165 and SDF-1 in coronary artery spasm. Eur Rev Med Pharmacol Sci. 2018;22(9): 2767-77.

29. Chan KH, Simpson PJ, Yong AS, Dunn LL, Chawantanpipat C, Hsu C, Yu Y, Keech AC, Celermajer DS, Ng MK. The relationship between endothelial 
progenitor cell populations and epicardial and microvascular coronary disease-a cellular, angiographic and physiologic study. PLoS One. 2014;9(4): e93980.

30. Yang SW, Hennessy RR, Khosla S, Lennon R, Loeffler D, Sun T, Liu Z, Park KH, Wang FL, Lerman LO, et al. Circulating osteogenic endothelial progenitor cell counts: new biomarker for the severity of coronary artery disease. Int J Cardiol. 2017;227:833-9.

31. Song ZY, Wang F, Cui SX, Gao ZH, Qu XJ. CXCR7/CXCR4 heterodimerinduced histone demethylation: a new mechanism of colorectal tumorigenesis. Oncogene. 2019;38(9):1560-75.

32. Dai X, Yan X, Zeng J, Chen J, Wang Y, Chen J, Li Y, Barati MT, Wintergerst KA, Pan K, et al. Elevating CXCR7 improves Angiogenic function of EPCs via Akt/GSK-3beta/Fyn-mediated Nrf2 activation in diabetic limb ischemia. Circ Res. 2017;120(5):e7-e23.

33. Zhang XY, Su C, Cao Z, Xu SY, Xia WH, Xie WL, Chen L, Yu BB, Zhang B, Wang $Y$, et al. CXCR7 upregulation is required for early endothelial progenitor cell-mediated endothelial repair in patients with hypertension. Hypertension. 2014;63(2):383-9.

34. Hu SC, Yu H, Yen FL, Chen GS, Lan CC. CXCR7 expression correlates with tumor depth in cutaneous squamous cell carcinoma skin lesions and promotes tumor cell survival through ERK activation. Exp Dermatol. 2014; 23(12):902-8.

35. Wang P, Zhang H, Li Z, Liu X, Jin Y, Lei M, Jiao Z, Bi Y, Guo W. Low-dose radiation promotes the proliferation and migration of AGE-treated endothelial progenitor cells derived from bone marrow via activating SDF1/CXCR4/ERK signaling pathway. Radiat Res. 2019;191(6):518-26.

36. Yu H, Zhang L, Liu P. CXCR7 signaling induced epithelial-mesenchymal transition by AKT and ERK pathways in epithelial ovarian carcinomas. Tumour Biol. 2015;36(3):1679-83.

37. Yang Z, Xia WH, Zhang YY, Xu SY, Liu X, Zhang XY, Yu BB, Qiu YX, Tao J. Shear stress-induced activation of Tie2-dependent signaling pathway enhances reendothelialization capacity of early endothelial progenitor cells. J Mol Cell Cardiol. 2012;52(5):1155-63.

38. Xu BJ, Chen J, Chen X, Liu XW, Fang S, Shu Q, Hu L, Shi SS, Du LZ, Tan LH. High shear stress-induced pulmonary hypertension alleviated by endothelial progenitor cells independent of autophagy. World J Pediatr. 2015;11(2):1716.

39. Montenegro FS, Correia M, Muccillo F, Souza E, Silva CG, De Lorenzo A. Associations between endothelial progenitor cells, clinical characteristics and coronary restenosis in patients undergoing percutaneous coronaryartery intervention. BMC Res Notes. 2018;8:11.

40. Xu WY, Tang WL, Yuan M, Sun Y, Xu F, Peng F. The adverse events rate of endothelial progenitor cell capturing stent in the treatment of CAD patients. Comb Chem High Throughput Screen. 2018;21(10):725-33.

\section{Publisher's Note}

Springer Nature remains neutral with regard to jurisdictional claims in published maps and institutional affiliations.

Ready to submit your research? Choose BMC and benefit from:

- fast, convenient online submission

- thorough peer review by experienced researchers in your field

- rapid publication on acceptance

- support for research data, including large and complex data types

- gold Open Access which fosters wider collaboration and increased citations

- maximum visibility for your research: over $100 \mathrm{M}$ website views per year

At $\mathrm{BMC}$, research is always in progress.

Learn more biomedcentral.com/submissions 